\title{
Seebeck effect in PbTe films and EuTe/PbTe superlattices
}

\author{
Akihiro Ishida, ${ }^{1, a)}$ Tomohiro Yamada, ${ }^{1}$ Daoshe Cao, ${ }^{1}$ Yoku Inoue, ${ }^{1}$ Martin Veis, ${ }^{2}$ \\ and Takuji Kita ${ }^{3}$ \\ ${ }^{1}$ Department of Electrical and Electronic Engineering, Shizuoka University, Johoku 3-5-1, Hamamatsu \\ 432-8561, Japan \\ ${ }^{2}$ Faculty of Mathematics and Physics, Charles University of Prague, Ke Karlovu 5, 12116 Prague 2, \\ Czech Republic \\ ${ }^{3}$ Toyota Motor Co., Mishuku 1200, Susono 410-1193, Japan
}

(Received 28 April 2009; accepted 20 June 2009; published online 28 July 2009)

\begin{abstract}
Theoretical calculations of the Seebeck coefficients of bulk PbTe and PbTe based superlattices were described in the framework of Boltzmann equation, taking into account temperature dependent band gaps, nonparabolicity, and anisotropy of effective masses. It is shown that the temperature gradient along the superlattice layer works more effectively on the enhancement of the thermoelectric figure of merit than the temperature gradient normal to the superlattice layer. Calculated Seebeck coefficients were compared to the experimental values for $n$-type $\mathrm{PbTe}, p$-type $\mathrm{PbTe}$, and EuTe/PbTe superlattices. The Seebeck coefficient of $p$-type $\mathrm{PbTe}$ was higher than that of $n$-type $\mathrm{PbTe}$. The relatively high Seebeck coefficient is explained by the contribution from other extrema in the valence band. The EuTe/PbTe [001] superlattice shows higher Seebeck coefficients than PbTe bulk owing to the large density of states. (C) 2009 American Institute of Physics.
\end{abstract}

[DOI: $10.1063 / 1.3182804]$

\section{INTRODUCTION}

Lead-salt IV-VI semiconductors have high thermoelectric figures of merits $Z=S^{2} \sigma / \kappa$ owing to the relatively high carrier mobility and low thermal conductivity. The figure of merit $Z$ increases also proportionally to the Seebeck coefficient squared, and a high Seebeck coefficient is very effective to increase the value of $Z$. Quantum well or superlattice (SL) structures are effective to decrease thermal conductivity. Enhancements of the Seebeck coefficient have been also reported in SL and quantum dot structures. ${ }^{1-3}$ In the discussion of the Seebeck coefficient, the Mott equation shown below is often used, ${ }^{4}$

$$
\begin{aligned}
S & =-\frac{\pi^{2} k^{2} T}{3 e}\left[\frac{\partial \ln \sigma(E)}{\partial E}\right]_{E=E_{F}} \\
& \approx-\frac{\pi^{2} k^{2} T}{3 e}\left[\frac{\partial \ln D(E)}{\partial E}\right]_{E=E_{F}},
\end{aligned}
$$

where $\sigma(E)$ is the energy dependent conductivity and $D(E)$ corresponds to the density of states. This equation is applicable for metals in which the density of states varies slowly compared to the Fermi-Dirac distribution. Thus this equation is not suitable for the quantitative discussion of the Seebeck coefficients for SLs and nondegenerate semiconductors. Recently we observed an enhancement of Seebeck coefficients in $\mathrm{EuTe} / \mathrm{PbTe} \mathrm{SLs}$ and explained the enhancement through the semiconductor theory based on Boltzmann's equation. In this paper, we describe the theoretical background more deeply and discuss the experimental Seebeck coefficients of $n$-type $\mathrm{PbTe}, p$-type $\mathrm{PbTe}$, and $n$-type EuTe/PbTe SLs.

\footnotetext{
${ }^{a)}$ Electronic mail: tdaishi@ipc.shizuoka.ac.jp.
}

\section{THEORETICAL CALCULATION OF SEEBECK COEFFICIENTS}

\section{A. Seebeck coefficient of bulk and SL materials}

The Seebeck coefficient of $n$-type semiconductor is given by

$$
S=\frac{1}{e} \frac{d E_{F}}{d T}=-\frac{1}{e} \frac{d\left(E_{C}-E_{F}\right)}{d T}+\frac{1}{e} \frac{d E_{C}}{d T},
$$

where $E_{F}$ and $E_{C}$ are the Fermi level and conduction band edge energy, respectively. The first term on the right side of the equation is determined by the density of states, and the second term, which we denote as kinetic part, is determined by the balance between drift and thermal diffusion currents. The second term is calculated from the Boltzmann equation. This equation is expressed as

$$
-\frac{f-f_{0}}{\tau}=-\hbar^{-1} \nabla E(\mathbf{k}) \cdot \nabla_{\mathbf{k}} f+\mathbf{v} \cdot \nabla f,
$$

where $f_{0}$ is the Fermi-Dirac distribution, $f$ is a nonequilibrium distribution, $E(\mathbf{k})$ is the energy of the electron state at the wavenumber $\mathbf{k}$, and $\mathbf{v}$ is the velocity of the electron. If we consider only the first term of Eq. (3), the drift current $J_{\text {drift }}$ is derived as

$$
\begin{aligned}
J_{\text {drift }} & =\sum_{V, m} \frac{1}{4 \pi^{3}} \int\left(-e v_{X}\right) f d^{3} k \\
& =\frac{e}{4 \pi^{3} k T} \sum_{V, m} \int \tau v_{X}^{2} f_{0}\left(1-f_{0}\right) \frac{d E(\mathbf{k})}{d X} d^{3} k,
\end{aligned}
$$

where we took the $X$ direction along the temperature gradient or electric field, while $v_{X}$ is the electron velocity along the temperature gradient, and the summation is taken for all valleys $V$ and for all subbands $m$ in the SL. In bulk semiconductor, the summation is taken for all valleys $V$. Thermal 
diffusion current is calculated from the second term of Eq. (3) as

$$
\begin{aligned}
J_{\text {thermal }}= & \frac{e}{4 \pi^{3} k T} \frac{d T}{d X} \sum_{V, m} \int \tau v_{X}^{2} f_{0}\left(1-f_{0}\right)\left\{\frac{E(\mathbf{k})-E_{F}}{T}\right. \\
& \left.-\frac{d\left[E(\mathbf{k})-E_{F}\right]}{d T}\right\} d^{3} k .
\end{aligned}
$$

The Seebeck coefficient is determined by the condition $J_{\text {drift }}+J_{\text {thermal }}=0$, and the following equation for the kinetic term is obtained:

$$
\begin{aligned}
\frac{1}{e} \frac{d E_{C}}{d T}= & \frac{1}{e} \frac{d\left(E_{C}-E_{F}\right)}{d T} \\
& -\frac{1}{e} \frac{\sum_{V, m} \int \tau v_{X}^{2} f_{0}\left(1-f_{0}\right) \frac{E(\mathbf{k})-E_{F}}{T} d^{3} k}{\sum_{V, m} \int \tau v_{X}^{2} f_{0}\left(1-f_{0}\right) d^{3} k} .
\end{aligned}
$$

With respect to Eq. (2), the Seebeck coefficient is given by

$$
S=-\frac{1}{e} \frac{\sum_{V, m} \int \tau v_{X}^{2} f_{0}\left(1-f_{0}\right) \frac{E(\mathbf{k})-E_{F}}{T} d^{3} k}{\sum_{V, m} \int \tau v_{X}^{2} f_{0}\left(1-f_{0}\right) d^{3} k} .
$$

Conduction band edge energy $E_{C}$ was eliminated in Eq. (6). Thus Eq. (6) can be used also for $p$-type materials or materials that include both electrons and holes such as narrow gap semiconductors and semimetals if we consider the contributions of all bands in the summation.
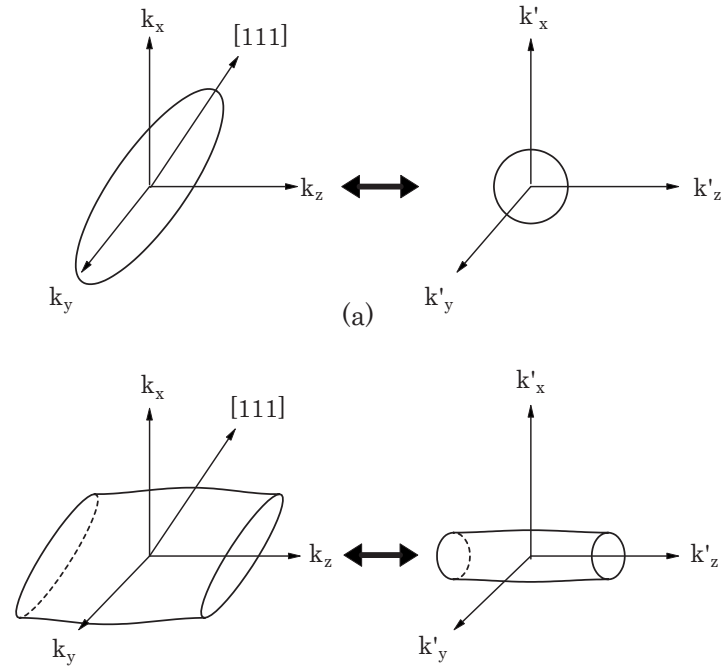

(b)

FIG. 1. Coordinate transformation used in this calculation (Ref. 5). Constant energy surfaces for the bulk and SL before and after transformation are shown in (a) and (b), respectively.

\section{B. Anisotropic semiconductors and SLs}

PbTe crystallizes in the rocksalt structure and has a many-valley direct gap at $L$-points of Brillouin zone with a large effective mass anisotropy of $m_{l} / m_{t}=10$. The calculation of the Seebeck coefficients for the semiconductors and SLs with the large effective mass anisotropy is somewhat complicated. However, the calculation becomes simple by introducing a coordinate transformation shown in Fig. 1, where the constant energy surface becomes a sphere keeping the SL direction in the same direction. ${ }^{5}$ The $E$-k relationship of the $L$-point valley is written as $\Sigma \hbar^{2} k_{i} k_{j} / 2 m_{i j}=E\left(1+E / E_{g}\right)$, where the effective mass tensor is given by

$$
\left[\begin{array}{ccc}
\frac{1}{m_{x x}} & \frac{1}{m_{x y}} & \frac{1}{m_{x z}} \\
\frac{1}{m_{y x}} & \frac{1}{m_{y y}} & \frac{1}{m_{y z}} \\
\frac{1}{m_{z x}} & \frac{1}{m_{z y}} & \frac{1}{m_{z z}}
\end{array}\right]=\left[\begin{array}{ccc}
\frac{\sin ^{2} \theta}{m_{l}}+\frac{\cos ^{2} \theta}{m_{t}} & 0 & \left(\frac{1}{m_{l}}-\frac{1}{m_{t}}\right) \sin \theta \cos \theta \\
0 & \frac{1}{m_{t}} & 0 \\
\left(\frac{1}{m_{l}}-\frac{1}{m_{t}}\right) \sin \theta \cos \theta & 0 & \frac{\cos ^{2} \theta}{m_{l}}+\frac{\sin ^{2} \theta}{m_{t}}
\end{array}\right] .
$$

Here, we took the SL direction in the $z$ direction and selected the $x$ direction so that the longitudinal axis of the ellipsoid lies in the $k_{z}-k_{x}$ plane. We used a coordinate transformation indicated below, where the constant energy surface becomes the sphere with an electron effective mass $m_{t}$.

$$
\left[\begin{array}{l}
k_{x}^{\prime} \\
k_{y}^{\prime} \\
k_{z}^{\prime}
\end{array}\right]=\left[\begin{array}{ccc}
\sqrt{\frac{m_{z z}}{m_{l}}} & 0 & 0 \\
0 & 1 & 0 \\
\frac{\sqrt{m_{t} m_{z z}}}{m_{z x}} & 0 & \sqrt{\frac{m_{t}}{m_{z z}}}
\end{array}\right]\left[\begin{array}{l}
k_{x} \\
k_{y} \\
k_{z}
\end{array}\right],
$$

$$
\left[\begin{array}{l}
x^{\prime} \\
y^{\prime} \\
z^{\prime}
\end{array}\right]=\left[\begin{array}{ccc}
\sqrt{\frac{m_{l}}{m_{z z}}} & 0 & -\frac{\sqrt{m_{l} m_{z z}}}{m_{z x}} \\
0 & 1 & 0 \\
0 & 0 & \sqrt{\frac{m_{z z}}{m_{t}}}
\end{array}\right]\left[\begin{array}{l}
x \\
y \\
z
\end{array}\right] .
$$

In this transformation, the product $\mathbf{k} \cdot \mathbf{r}$ is conserved and we can perform the integration in Eq. (7) simply in this new coordinate system if we consider that the $\mathbf{k}$-coordinate electron velocity $\mathbf{v}$ corresponding to the position $\left(k_{x}^{\prime}, k_{y}^{\prime}, k_{z}^{\prime}\right)$ is given by 


$$
\begin{aligned}
\mathbf{v}= & \frac{1}{\hbar} \nabla_{\mathbf{k}} E=\frac{1}{\hbar}\left[\begin{array}{c}
\frac{\partial E}{\partial k_{x}} \\
\frac{\partial E}{\partial k_{y}} \\
\frac{\partial E}{\partial k_{z}}
\end{array}\right]=\frac{1}{\hbar}\left[\begin{array}{ccc}
\sqrt{\frac{m_{z z}}{m_{l}}} & 0 & \frac{\sqrt{m_{t} m_{z z}}}{m_{z x}} \\
0 & 1 & 0 \\
0 & 0 & \sqrt{\frac{m_{t}}{m_{z z}}}
\end{array}\right] \\
& \times\left[\begin{array}{c}
\frac{\partial E}{\partial k_{x}^{\prime}} \\
\frac{\partial E}{\partial k_{y}^{\prime}} \\
\frac{\partial E}{\partial k_{z}^{\prime}}
\end{array}\right] .
\end{aligned}
$$

Thus the Seebeck coefficient for the SL is given by

$$
S_{\mathrm{SL}}=-\frac{1}{e} \frac{\sum_{V, m} \int_{-\frac{\pi}{D^{\prime}}}^{\frac{\pi}{D^{\prime}}} \int_{0}^{\infty} \int_{0}^{2 \pi} \pi v_{X}^{2} f_{0}\left(1-f_{0}\right) \frac{E-E_{F}}{T} k^{\prime 2} d \phi d k_{/ /}^{\prime} d k_{Z}^{\prime}}{\sum_{V, m} \int_{-\frac{\pi}{D^{\prime}}}^{\frac{\pi}{D^{\prime}}} \int_{0}^{\infty} \int_{0}^{2 \pi} \pi v_{X}^{2} f_{0}\left(1-f_{0}\right) k^{\prime 2} d \phi d k_{/ /}^{\prime} d k_{Z}^{\prime}},
$$

where $k_{\mu}^{\prime}$ is the wavenumber in $k_{x}^{\prime}-k_{y}^{\prime}$ plane and $D^{\prime}$ given by $\left(m_{z z} / m_{t}\right)^{1 / 2} D$ is the SL period in the $x^{\prime} y^{\prime} z^{\prime}$ coordinate. When we take the temperature gradient ( $X$ direction) in the $x-y$ plane with the angle $\alpha$ from the $x$ axis, the $v_{X}$ becomes

$$
v_{X}=\frac{1}{\hbar}\left(\sqrt{\frac{m_{z z}}{m_{l}}} \frac{\partial E}{\partial k_{x}^{\prime}}+\frac{\sqrt{m_{t} m_{z z}}}{m_{z x}} \frac{\partial E}{\partial k_{z}^{\prime}}\right) \cos \alpha+\frac{1}{\hbar} \frac{\partial E}{\partial k_{y}^{\prime}} \sin \alpha,
$$

and if the temperature gradient is the $z$ direction,

$$
v_{X}=\frac{1}{\hbar} \sqrt{\frac{m_{t}}{m_{z z}}} \frac{\partial E}{\partial k_{z}^{\prime}}=\frac{1}{\hbar} \frac{\partial E}{\partial k_{z}} .
$$

In bulk materials, we take the $z$ axis in the direction of the temperature gradient. Then the Seebeck coefficient is calculated simply by

$$
S_{\text {bulk }}=-\frac{1}{e} \frac{\sum_{V} \int_{0}^{\infty} \int_{0}^{\pi} \pi v_{X}^{2} f_{0}\left(1-f_{0}\right) \frac{E-E_{F}}{T} k^{\prime 2} d \theta d k^{\prime}}{\sum_{V} \int_{0}^{\infty} \int_{0}^{\pi} \tau v_{X}^{2} f_{0}\left(1-f_{0}\right) k^{\prime 2} d \theta d k^{\prime}},
$$

where the $v_{X}$ is given by

$$
v_{X}=\frac{1}{\hbar} \sqrt{\frac{m_{t}}{m_{z z}}} \frac{\partial E}{\partial k_{z}^{\prime}}=\frac{\hbar k^{\prime} \cos \theta}{\sqrt{m_{z z} m_{t}}\left(1+\frac{2 E}{E_{g}}\right)} .
$$

In the calculation, we considered $m_{l}=0.4 m_{0}$ and $m_{t}$ $=0.04 m_{0}$ for $\mathrm{PbTe}$, assuming that the band edge effective masses are proportional to the band gap. The conduction band offset of the EuTe/PbTe heterojunction was assumed to be $1000 \mathrm{meV}$ with the parameter used in Ref. 6. Energy dependent relaxation time $\tau \propto E^{-1 / 2}$ was used for the calculation assuming dominant acoustic-phonon scattering. Figure 2 shows the theoretical dependence of the Seebeck coefficients

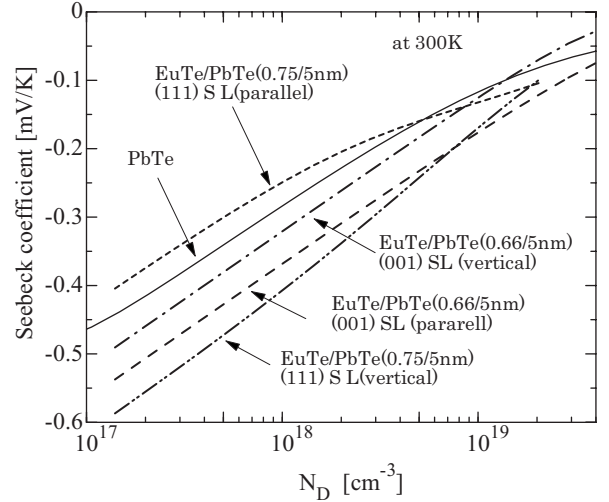

FIG. 2. The dependence of the Seebeck coefficients on donor concentration $N_{\mathrm{D}}$ calculated for the PbTe film, EuTe/PbTe $0.66 \mathrm{~nm} / 5 \mathrm{~nm}$ [001] SL, and $\mathrm{EuTe} / \mathrm{PbTe} 0.75 \mathrm{~nm} / 5 \mathrm{~nm}$ [111] SL.

on donor concentration $N_{\mathrm{D}}$ for the $\mathrm{PbTe}$ film, EuTe/PbTe $0.66 \mathrm{~nm} / 5 \mathrm{~nm}$ [001] SL, and EuTe/PbTe $0.75 \mathrm{~nm} / 5 \mathrm{~nm}$ [111] SL. Seebeck coefficients parallel and perpendicular to the SL direction were calculated, and it was assumed that all the donors make electrons in the conduction band. The effect of impurity levels or impurity band on the Seebeck effect was not considered here. The Seebeck coefficient of EuTe/PbTe [001] SL parallel to the SL layer (denoted by parallel) increases owing to the increase in the density of states, while the parallel Seebeck coefficient of EuTe/PbTe [111] SL decreases owing to the decrease in the kinetic part of Eq. (2), as discussed in Ref. 3. The Seebeck coefficient normal to the SL layer becomes smaller than that parallel to the layer for the EuTe/PbTe [001] SL owing to the modulation of $v_{X}$ due to the SL potential. On the other hand, the parallel Seebeck coefficient for the EuTe/PbTe [111] SL becomes larger than that of the [001] SL because many of the carriers are distributed in the longitudinal valley, where they do not contribute to the conductivity perpendicular to the layer. Therefore, a relatively small number of carriers in the oblique valleys give such high Seebeck coefficient. If we compare the Seebeck coefficient as a function of the carrier concentration in the oblique valleys, Seebeck coefficients for parallel and perpendicular directions become comparable. Anyway, since the

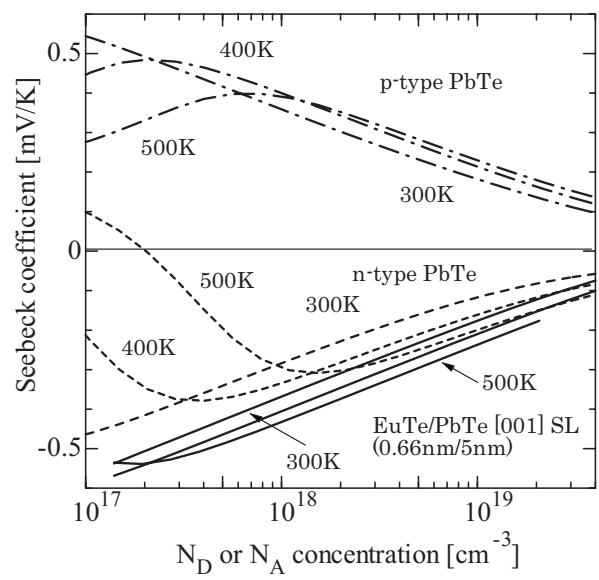

FIG. 3. The dependence of in-plane Seebeck coefficients on impurity concentration for $n$-type PbTe, $p$-type PbTe, and $n$-type EuTe/PbTe [001] SL with the parameter of temperature. 

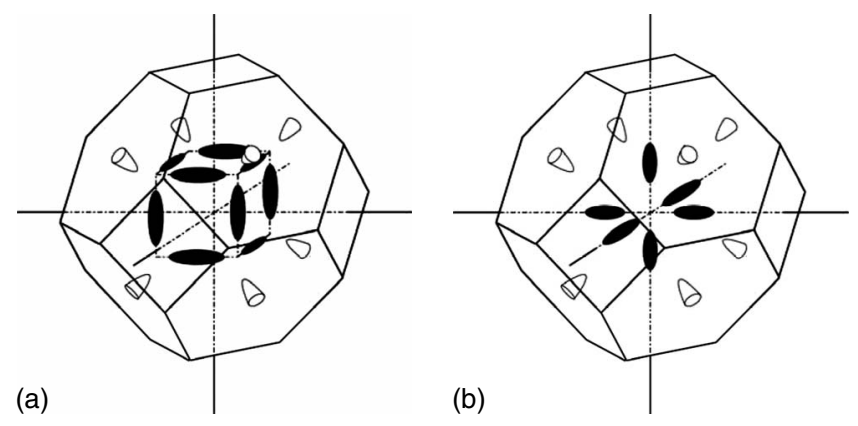

FIG. 4. Possible constant energy surfaces of PbTe valence band in which shadowed second ellipsoids exist along the (a) $\Sigma$ axes and (b) $\triangle$ axes, respectively.

conductivity along the SL layer is much larger than that perpendicular to the layer, thermoelectric figure of merit $Z T$ along the SL layer becomes higher than that perpendicular to the layer.

Figure 3 shows the dependence of the Seebeck coefficients on the impurity concentration for $n$-type $\mathrm{PbTe}, p$-type $\mathrm{PbTe}$, and $n$-type EuTe/PbTe [001] SL with the parameter of temperature. The absolute values of the Seebeck coefficients increase with temperature, but the significant increase in minority carriers decreases the Seebeck coefficient in the low impurity region. Since PbTe has almost same effective masses in the conduction and valence band at $L$-point of the Brillouin zone, the absolute value of Seebeck coefficient becomes almost the same if we consider only $L$-point carriers. $\mathrm{In} \mathrm{PbTe}$, there are certain indirect band extrema near the top of the valence band, and it is known that the PbTe becomes indirect gap semiconductor above $420 \mathrm{~K}$. Possible positions of the extrema are along $\Sigma$ axes $(\langle 110\rangle$ directions) and $\Delta$ axes $(\langle 100\rangle$ directions) from the band structure calculation in empirical pseudopotential method. ${ }^{7}$ Sitter et al. ${ }^{8}$ showed the main axes of the second constant energy ellipsoids direct $\langle 100\rangle$ with the effective mass anisotropy approximately ten from the magnetoresistance measurement and proposed $12 \Sigma$ ellipsoids with the main axes along the $\langle 100\rangle$ directions. ${ }^{8}$ Figures 4(a) and 4(b) show the possible constant energy surfaces of the valence band. We assumed that the second valence band maxima are $50 \mathrm{meV}$ below the $L$-point maxima at $300 \mathrm{~K}$, and the valence band maxima increase relatively to the $L$-point maxima as $0.4 \mathrm{meV} / \mathrm{K}$ in the calculation of $p$-type PbTe in Fig. 3 using the model in Fig. 4(a). We used $m_{l}=0.49 m_{0}$ and $m_{t}=0.035 m_{0}$ for the second ellipsoid as was used in the calculation in Ref. 8.

\section{EXPERIMENTAL SEEBECK COEFFICIENTS}

PbTe films were prepared on $\mathrm{BaF}_{2}(111)$ substrates and EuTe/PbTe short-period SLs with $5 \mathrm{~nm}$ PbTe and 1-2 ML (monolayer) EuTe were prepared on $\mathrm{KCl}$ (001) substrates by hot wall epitaxy using $\mathrm{PbTe}, \mathrm{Eu}$, and Te as source materials. The SL structure was confirmed by $\mathrm{x}$-ray diffraction, and the quality of the SL structure prepared on $\mathrm{KCl}(001)$ substrate has also been ascertained by strong intersubband absorption in the EuTe/PbTe double-well SL. ${ }^{6}$ The substrate temperature during the growth was $280{ }^{\circ} \mathrm{C}$, and the total thicknesses of the SLs were 1.5-4 $\mu \mathrm{m}$. The film thickness was calculated from the optical transmission spectrum measured by Fourier transform infrared spectroscopy. The carrier concentration was obtained from Hall measurement. The Seebeck coefficient was measured with ZEM-2 (ULVAC-Riko). The measurement was performed in a helium atmosphere to decrease the temperature error. The temperature difference between two thermocouples was applied in $5 \mathrm{~K}$. The Seebeck coefficient was measured within the error of $10 \%$.

Table I shows the electrical properties of the PbTe films and $\mathrm{EuTe} / \mathrm{PbTe} \mathrm{SLs}$ with the electrical property of $\mathrm{PbEuTe} /$ $\mathrm{PbTe} \mathrm{SL}$ for comparison. $\mathrm{PbTe}$ has carrier mobilities of $1700 \mathrm{~cm}^{2} / \mathrm{V} \mathrm{s}$ for $n$-type and $840 \mathrm{~cm}^{2} / \mathrm{V} \mathrm{s}$ for $p$-type, respectively. ${ }^{9}$ Our data are comparable to the reported ones. In $\mathrm{Pb}_{1-x} \mathrm{Eu}_{x} \mathrm{Te}(x \approx 0.05)$ ternary alloy, the typical carrier mobility is around $100 \mathrm{~cm}^{2} / \mathrm{V} \mathrm{s}$ because the Eu atom works as strong scattering center. This effect works also for the $\mathrm{PbEuTe} / \mathrm{PbTe}$ and $\mathrm{EuTe} / \mathrm{PbTe}$ SLs through the interdiffusion of Eu or interface roughness, and the carrier mobility decreases somewhat in the SLs, as shown in Table I.

Figure 5 shows the dependence of the Seebeck coefficients for the $p$-type $\mathrm{PbTe}, n$-type $\mathrm{PbTe}$, and $n$-type EuTe/ $\mathrm{PbTe}$ SLs. Excellent agreement between experimental and theoretical Seebeck coefficients were obtained for $n$-type $\mathrm{PbTe}$ films and $n$-type EuTe/PbTe SLs, indicating considerably high Seebeck coefficient in the EuTe/PbTe SLs compared to the PbTe films. The Seebeck coefficient of the $p$-type $\mathrm{PbTe}$ film was higher than that of the $n$-type film, which is considered to be due to the second valence band maxima. Among three theoretical lines in $p$-type region, the dashed line shows the values that assumed no second valley effect, the dashed-dotted line was assumed to be the model in Fig. 4(a), and the dashed-double dotted line was assumed to be the model in Fig. 4(b). Good agreement was obtained in

TABLE I. Typical electrical properties of the PbTe films and EuTe/PbTe SLs with the electrical property of $\mathrm{PbEuTe} / \mathrm{PbTe} \mathrm{SL}$ for comparison.

\begin{tabular}{lcccccc}
\hline \hline Sample & $\begin{array}{c}\text { Structure } \\
(\mathrm{nm} / \mathrm{nm})\end{array}$ & $\begin{array}{c}\text { Resistivity } \\
(\Omega \mathrm{cm})\end{array}$ & $\begin{array}{c}\text { Mobility } \\
\left(\mathrm{cm}^{2} / \mathrm{V} \mathrm{s}\right)\end{array}$ & Carrier type & $\begin{array}{c}\text { Carrier con. } \\
\left(\mathrm{cm}^{-3}\right)\end{array}$ & Dopant \\
\hline $\mathrm{PbTe}$ & & 0.00346 & 795 & $p$ & $2.3 \times 10^{18}$ & $\mathrm{Tl}$ \\
$\mathrm{PbTe}$ & & 0.00284 & 1410 & $n$ & $1.6 \times 10^{18}$ & $\mathrm{Bi}$ \\
$\mathrm{PbEuTe} / \mathrm{PbTe}$ & $10 / 10$ & 0.00156 & 540 & $n$ & $7.4 \times 10^{18}$ & $\mathrm{Bi}$ \\
$\mathrm{EuTe} / \mathrm{PbTe}$ & $0.3 / 11$ & 0.0042 & 880 & $n$ & $1.7 \times 10^{18}$ & $\mathrm{Bi}$ \\
$\mathrm{EuTe} / \mathrm{PbTe}$ & $0.3 / 6.2$ & 0.0028 & 446 & $n$ & $5.0 \times 10^{18}$ & $\mathrm{Bi}$ \\
\hline \hline
\end{tabular}




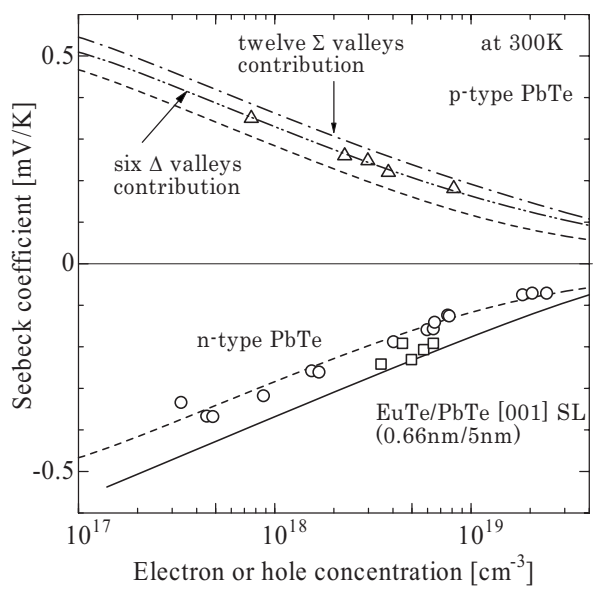

FIG. 5. Comparison between experimental and theoretical Seebeck coefficients for $n$-type $\mathrm{PbTe}, p$-type $\mathrm{PbTe}$, and $n$-type EuTe/PbTe [001] SLs at room temperature. The dots $\bigcirc, \square$, and $\triangle$ show experimental values for $n$-type $\mathrm{PbTe}, n$-type EuTe/PbTe SL, and $p$-type $\mathrm{PbTe}$, respectively. The solid line shows theoretical values for EuTe/PbTe $(0.66 \mathrm{~nm} / 5 \mathrm{~nm}) \mathrm{SL}$. The dashed line in $n$-type region shows theoretical values for $n$-type $\mathrm{PbTe}$, and dashed line in $p$-type region shows theoretical values for $p$-type $\mathrm{PbTe}$ where no indirect valley effect was considered. The dashed-doted line and dasheddouble dotted line show theoretical lines where $12 \Sigma$ valleys and $6 \Delta$ valleys were assumed, respectively. Good agreement between theoretical and experimental Seebeck coefficients were obtained, and significant increase in Seebeck coefficients compared to PbTe films was obtained in the EuTe/PbTe $(0.66 \mathrm{~nm} / 5 \mathrm{~nm})$ [001] SLs. The Seebeck coefficient of $p$-type PbTe becomes higher than that of $n$-type $\mathrm{PbTe}$ owing to the contribution of second valence band maxima.

the model in Fig. 4(b) with the effective masses of $m_{l}$ $=0.49 m_{0}$ and $m_{t}=0.035 m_{0}$. To fit the theoretical line to the experimental values for the model in Fig. 4(a), we have to assume very small effective masses as high as $m_{l}=0.15 m_{0}$ and $m_{t}=0.015 m_{0}$ for the second ellipsoids, which do not agree to the pseudopotential calculation in Ref. 7. Anyway, the relatively large Seebeck coefficient in $p$-type $\mathrm{PbTe}$ was well explained by the effect of second valence band maxima.

\section{SUMMARY}

Theoretical calculation of the Seebeck coefficient was presented in this paper, taking into account nonparabolicity and anisotropy of effective masses. The dependence of the Seebeck coefficients on the carrier concentration was calculated for $n$-type $\mathrm{PbTe}, p$-type $\mathrm{PbTe}$, and EuTe/PbTe SLs. The Seebeck coefficient of $p$-type PbTe becomes higher owing to the contribution from second valence band maxima. In [001] $\mathrm{EuTe} / \mathrm{PbTe}$ SL, the Seebeck coefficient along SL layer becomes higher than that perpendicular to the layer, and higher thermoelectric figure of merit is expected in the layer plane. In EuTe/PbTe [111] SL, the Seebeck coefficient perpendicular to the layer increases considerably compared to that along the layer. This is due to the relatively small carrier concentration in the oblique valleys which determines the Seebeck coefficient. Seebeck coefficients for $n$-type $\mathrm{PbTe}, p$-type $\mathrm{PbTe}$, and $n$-type EuTe/PbTe SLs were measured and good agreement between theoretical and experimental values was obtained.

${ }^{1}$ L. D. Hicks, T. C. Harman, X. Sun, and M. S. Dresselhaus, Phys. Rev. B 53, R10493 (1996).

${ }^{2}$ T. C. Harman, P. J. Taylor, D. L. Spears, and M. P. Walsh, J. Electron. Mater. 29, L1 (2000).

${ }^{3}$ A. Ishida, D. Cao, S. Morioka, M. Veis, Y. Inoue, and T. Kita, Appl. Phys. Lett. 92, 182105 (2008)

${ }^{4}$ J. P. Heremans, V. Jovovic, E. S. Toberer, A. Saramat, K. Kurosaki, A. Charoenphakdee, S. Yamanaka, and G. J. Snyder, Science 321, 554 (2008).

${ }^{5}$ A. Ishida, D. Cao, S. Morioka, Y. Inoue, and T. Kita, J. Electron. Mater. 38, 940 (2009).

${ }^{6}$ A. Ishida, M. Veis, and Y. Inoue, Jpn. J. Appl. Phys., Part 2 46, L281 (2007).

${ }^{7}$ G. Martinez, M. Schlüter, and M. L. Cohen, Phys. Rev. B 11, 651 (1975).

${ }^{8}$ H. Sitter, K. Lischka, and H. Heinrich, Phys. Rev. B 16, 680 (1977).

${ }^{9}$ Y. I. Ravich, Lead Chalcogenides: Physics and Applications (Taylor \& Francis, New York, 2003), Ch.ap. 1, p. 20. 